\title{
Fever in a Man with HIV: An Unusual Case of an Immune System Gone Wrong
}

Emily Sutton, MD, Aishah Ali, MD

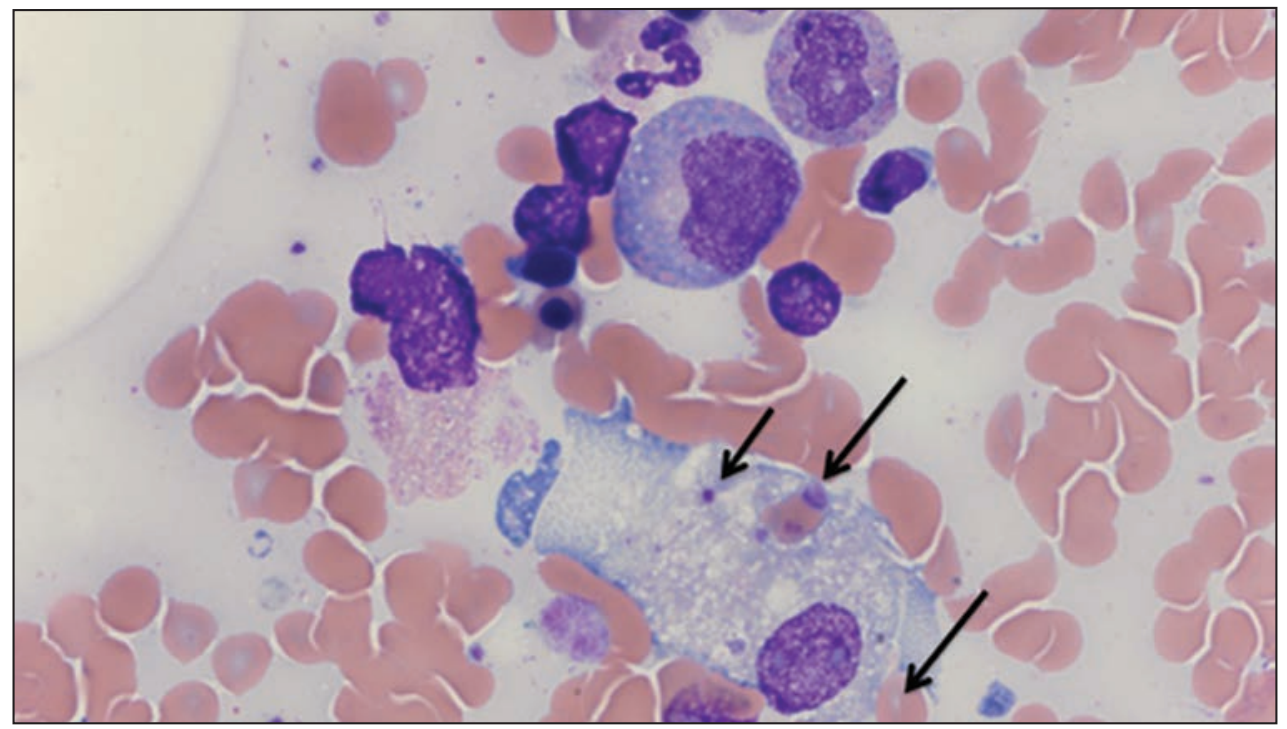

Figure 1: The patient's bone marrow biopsy demonstrating hemophagocytosis of platelets (short arrow) and erythrocytes (long arrows).

\section{INTRODUCTION}

Hemophagocytic lymphohistiocytosis (HLH) is a rare syndrome of immune dysregulation that is often recognized as secondary to an underlying immune activating state, such as malignancy, rheumatologic disorders, and infections. This case highlights an association between HLH and human immunodeficiency virus (HIV) infection. Although HLH is a rare complication of HIV, it presents a difficult challenge for treatment. Without treatment, HLH is invariably fatal, but the consequence of the immunosuppressive treatment regimen in the setting of an underlying opportunistic infection can also have fatal outcomes.

\section{CASE PRESENTATION}

A 38 year-old man with a history of HIV infection with a CD4 lymphocyte count of 2 cells $/ \mathrm{mm}^{3}$ presented with fevers two weeks after starting antiretroviral therapy (ART). His initial extensive infectious workup was negative, including cryptococcal antigen in the serum and cerebrospinal fluid. His liver enzymes then began to rise, prompting concern for drug toxicity. At this time, ART and trimethoprim/sulfamethoxazole were discontinued, but he continued to have high-grade fevers as well as hypotension, pancytopenia and hypofibrinogenemia and was transferred to the medical intensive care unit (MICU). On admission to the MICU, he was febrile with a temperature of $103.6^{\circ} \mathrm{F}$, hypotensive with a blood pressure of $78 / 37 \mathrm{mmHg}$, tachycardic with a heart rate of 127 beats per minute, tachypneic with a respiratory rate of 26 breaths per minute, and had an oxygen saturation of $97 \%$ on room air. On exam, he was thin, in moderate distress, and lethargic. He had no thrush or nuchal rigidity. He had cervical lymphadenopathy and hyperpigmented maculopapular rashes on his lower extremities; otherwise his exam was unremarkable.

\section{DIFFERENTIAL DIAGNOSIS}

The differential diagnosis included opportunistic infections given his CD4 count of 2 cells $/ \mathrm{mm}^{3}$, immune reconstitution inflammatory syndrome (IRIS) given his 


\begin{tabular}{l} 
Table 1: Diagnostic Criteria for HLH3 \\
Diagnosis of HLH requires \\
Molecular diagnosis consistent with $\mathrm{HLH}$ \\
-OR- \\
Fulfillment of 5 of the 8 criteria listed below: \\
1. Fever $\geq 38.5^{\mathrm{c}}$ \\
2. Splenomegaly \\
3. Cytopenias (affecting at least 2 of 3 lineages \\
Hemoglobin $<9 \mathrm{~g} / \mathrm{dL}$ \\
Platelets $<100 \times 10^{3} / \mathrm{mL}$ \\
Neutrophils $<1 \times 10^{3} / \mathrm{mL}$ ) \\
4. Hypertriglyceridemia (fasting $>265 \mathrm{mg} / \mathrm{dL}$ ) and/or \\
hypofibrinogenemia (< $150 \mathrm{mg} / \mathrm{dL}$ ) \\
5. Hemophagocytosis in bone marrow, spleen, lymph \\
nodes, or liver \\
6. Low or absent $\mathrm{NK}$-cell activity \\
7. Ferritin $>500 \mathrm{ng} / \mathrm{mL}$ \\
\hline
\end{tabular}

recent initiation of ART, drug reaction or toxicity, and hemophagocytic lymphohistiocytosis $(H L H)$. Further laboratory studies revealed an elevated ferritin level of $83,987 \mathrm{ng} / \mathrm{mL}$ (normal range $=30-400$ ), which was highly suspicious for HLH. The diagnosis was confirmed with a bone marrow biopsy revealing hemophagocytosis (Figure 1) as well as an elevated interleukin 2 receptor (IL2R) level of $9020 \mathrm{pg} / \mathrm{mL}$ (normal range $=0-1033$ ).

\section{OUTCOME AND FOLLOW-UP}

The patient was treated with intravenous immunoglobulin, high dose dexamethasone, and anakinra, an IL1R inhibitor, resulting in cessation of his fevers, resolution of his respiratory distress, and improvement in his cell lines. He was transferred back to the floors with plans to restart ART. As no underlying infection had been found, his HLH was thought to be secondary to his HIV infection. However, three days later, he became acutely short of breath and hypotensive and was found to have Cryptococcus neoformans fungemia, although his initial blood and cerebrospinal fluid fungal studies were negative. He was transferred back to the MICU, initiated on intravenous amphotericin B/flucytosine, and ART was discontinued. However, he continued to decompensate rapidly and expired from septic shock and acute pulmonary edema later that day.

\section{DISCUSSION}

Hemophagocytic lymphohistiocytosis $(H L H)$ is a severe and rapidly progressive disorder of immune activation and dysregulation that can occur as a familial disorder or, as is becoming increasingly recognized, secondary to a variety of underlying conditions. Secondary HLH occurs after strong immunologic activation, such as with severe infection, immunodeficiency, or underlying malignancy. In the past, HLH was also sometimes referred to as hemophagocytic syndrome. Another disorder on the spectrum of this disease state includes macrophage activation syndrome, which is a form of $\mathrm{HLH}$ associated with rheumatologic diseases. ${ }^{1}$ Although the immune cells in $\mathrm{HLH}$ are functionally normal, it is thought to be the result of proliferation of activated $T$ cells that go on to activate macrophages, as well as the lack of appropriate apoptosis of immunogenic cells. As these overly active macrophages and histiocytes proliferate and run rampant, they phagocytize other cells, including erythrocytes, leukocytes, and platelets, leading to the clinical symptoms. This highly stimulated immune system results in life-threatening cytokine storm and inflammatory reactions. ${ }^{1.2}$

As the clinical entity of HLH is a syndrome, it has features that can be seen in other clinical states, but it is the combination of findings that make the diagnosis likely. The diagnostic criteria for HLH include fever, splenomegaly, cytopenia of at least 2 of 3 cell lines, elevated ferritin, hypofibrinogenemia and/or hypertriglyceridemia, elevated CD 25 (IL-2 receptor), low or absent natural killer (NK) cells, and hemophagocytosis seen in the bone marrow, liver, spleen, or lymph nodes. Five of these eight criteria must be met in order to establish the diagnosis (Table 1). ${ }^{3}$ Notably, the criteria only require a ferritin level $>500 \mathrm{ng} / \mathrm{mL}$, but a level $>10,000 \mathrm{ng} / \mathrm{mL}$ is thought to be highly suspicious for $\mathrm{HLH}$, with a specificity of $96 \%{ }^{4}$ The ferritin level upon diagnosis has also been found to have prognostic value, and its decline correlates with response to treatment. The soluble IL-2 receptor was found to be a more sensitive marker than ferritin with a sensitivity of $93 \%$ and also carries prognostic implications. ${ }^{5}$ 
Without therapy, HLH is uniformly fatal. Treatment includes supportive care, immunomodulatory therapy, and treatment of any underlying condition. For the familial form of $\mathrm{HLH}$, the treatment backbone is an induction phase involving chemotherapy, typically etoposide, combined with immunotherapy, such as cyclosporine A and steroids. This is followed by a continuation phase that is to be maintained until stem cell transplant is available, as transplant is the only curative treatment for familial HLH. The treatment for secondary HLH is not as well defined as it can vary based on the associated disease. For underlying malignancies, the treatment approach is often similar to the familial form, with chemotherapy and immunotherapy. For the secondary forms associated with immunodeficiency or rheumatologic causes, the approach relies more heavily on immunomodulators, such as the IL-1 receptor antagonist anakinra. ${ }^{2}$

In the context of HIV, HLH has been reported to occur secondary to opportunistic infections, as well as from HIV infection itself, both during the acute seroconversion phase and the profoundly immunosuppressed state. ${ }^{6.7}$ Given the non-specific clinical manifestations of $\mathrm{HLH}$ and the otherwise heightened concern for opportunistic infections, one must have a high clinical suspicion for recognizing this disease in the setting of HIV. Treating HLH in the setting of severe immunosuppression from HIV presents a difficult challenge, as with this patient. As induction treatment for $\mathrm{HLH}$ requires high dose steroids and IV immunoglobulin to quell the life-threatening inflammatory response, underlying opportunistic infections may prove to be fatal if they go unrecognized. There are no current guidelines on antibiotic use in HIV patients with confirmed HLH, and it may be of value to treat these patients empirically for opportunistic infections in the setting of critical illness.

\section{KEY POINTS}

Patients presenting with a clinical picture of fever and highly elevated inflammatory markers should raise the suspicion for hemophagocytic lymphohistiocytosis $(H L H)$. Five of the eight criteria must be met for the diagnosis, but a ferritin level $>10,000 \mathrm{ng} / \mathrm{mL}$ is highly suspicious. HLH is fatal without treatment, but immunomodulatory therapy directed at HLH may be perilous in the setting of underlying infection. Currently, there are no guidelines on empiric antibiotic use for patients with HIV and HLH, but one must be vigilant in searching for underlying infections given the risk of the immunosuppressive regimen required to treat $\mathrm{HLH}$ in the setting of HIV.

\section{REFERENCES}

1. Jordan MB, Allen CE, Weitzman S, Filipovich AH, McClain KL. How I treat hemophagocytic lymphohistiocytosis. Blood. 2011; 118(15): 4041-52.

2. Mehta RS, Smith RE. Hemophagocytic lymphohistiocytosis (HLH): a review of the literature. Med Oncol. 2013; 30(4): 740.

3. Henter Jl, Horne A, Arico M, et al. HLH-2004: diagnostic and therapeutic guidelines for hemophagocytic lymphohistiocytosis. Pediatr Blood Cancer. 2007; 48(2): 124-131.

4. Tang $Y, X u X$. Advances in hemophagocytic lymphohistiocytosis: pathogenesis, early diagnosis/differential diagnosis, and treatment. Scientific World Journal. 2011; 11: 697-708.

5. Tothova Z, Berliner N. Hemophagocytic syndrome and critical illness: new insights into diagnosis and management. J Intesive Care Med. 2014 Jan 8 [Epub ahead of print]

6. Doyle T, Bhagani S, Cwynarski K. Haemophagocytic syndrome and HIV. Curr Opin Infect Dis. 2009; 22(1): 1-6.

7. Chen TL, Wont WW, Chiou TJ. Hemophagocytic syndrome: an unusual manifestation of acute human immunodeficiency virus infection. Int J Hematol. 2003; 78(5): 450-2. 